\title{
PRESENTACIÓN DE INFORMACIÓN FINANCIERA, UNA CARACTERÍSTICA DE MEJORA EN LAS ENTIDADES DEL SECTOR PÚBLICO ADOPTANTES DE NORMAS INTERNACIONALES
}

\author{
PRESENTATION FINANCIAL STATEMENTS, \\ AN OPPORTUNITY FOR IMPROVEMENT \\ IN PUBLIC ENTITIES ADOPTING \\ INTERNATIONAL STANDARDS
}

\author{
Leidy Katerine Rojas Molina ${ }^{1}$
}

\section{RESUMEN}

Las entidades del sector público en Colombia han experimentado importantes cambios respecto a la normatividad contable que deben aplicar y a los criterios de reconocimiento medición, revelación y presentación que deben seguir, la entrada en vigencia de la Resolución 533 de 2015, por la cual se realiza convergencia indirecta de las Normas internacionales de Contabilidad del Sector Publico (NICSP), emitidas por la IFAC, para las entidades de gobierno, busca como lo ha señalado dicha resolución, avanzar en materia de rendición de cuentas, transparencia y comparabilidad, fijando criterios uniformes para las entidades de gobierno.

$\mathrm{Al}$ analizar los criterios de presentación de la información contable que han aplicado las entidades gubernamentales, se evidencian falencias en la presentación de información financiera. Por ello, se busca identificar en qué aspectos se generan las principales diferencias frente a los criterios de presentación de la información contable establecidos por las NICSP, La metodología empleada permite comparar los criterios mínimos de presentación que deben considerar las entidades del sector público, una vez realicen la adopción completa de NICSP, frente a los criterios de presentación actuales de los Estados Financieros. Esta comparación se realizara empleando el índice sugerido por Pérez \& López, (2003), actualizado en esta investigación considerando los nuevos requerimientos de NICSP para las entidades de gobierno en Colombia.

Los resultados permiten identificar aspectos que afectan la presentación de la información contable en estas entidades, ya que en ciertos casos se observa información incompleta u omisión absoluta de algunos estados financieros, lo cual permite establecer factores de mejora sobre los que deben trabajar las entidades gubernamentales.

Palabras claves: Estados Financieros, NICSP, Presentación, Transparencia, Sector Público.

Recepción: 04/09/2018. Aprobación: 03/12/2018.

1 Fundación Universitaria San Mateo, Bogotá, Colombia, lkrojasm@unal.edu.co 


\section{ABSTRACT}

Public sector entities in Colombia have undergone significant changes regarding the accounting standards to be applied and the measurement, disclosure and presentation recognition criteria to be followed, the enforcement of Resolution 533 of 2015, for which indirect convergence of the International Public Sector Accounting Standards was achieved (IPSAS), issued by IFAC, for government entities, seeks as has been pointed out in this resolution, to advance in terms of accountability, transparency and comparability, establishing uniform criteria for government entities.

When analyzing the criteria for the presentation of accounting information that have been applied by government entities, there is a lack of presentation of financial information, as opposed to the presentation criteria established by the IPSAS. Therefore, it seeks to identify in certain aspects the main differences that are generated against the criteria for the presentation of accounting information, which should be considered by public entities belonging to the education sector. The methodology used allows comparing the minimum criteria for presentation that should be considered by public sector entities, once they complete the full adoption of IPSAS, against the current presentation criteria of the Financial Statements. This comparison will be made using the index suggested by Perez \& Lopez, (2003), updated in this research considering the new requirements of IPSAS for government entities in Colombia.

The results allow us to identify aspects that affect the presentation of accounting information in these entities, since in some cases incomplete information or absolute omission of some financial statements can be identified, which allows us to identify improvement factors on which government entities must work.

Keywords: IPSAS, Financial Statements, Disclosure, Public sector, Transparency.

\section{INTRODUCCIÓN}

Las entidades que aplican Normas Internacionales de Contabilidad para el Sector Publico (NICSP) en Colombia están conformadas por diversos sectores, que continuamente se ven afectados por investigaciones asociadas a malversación de fondos y falta de transparencia en la rendición de cuentas. Baracaldo (2013), establece conforme a la definición de la $\mathrm{OCDE}^{2}$ que la transparencia garantiza la revelación oportuna y precisa de todas las cuestiones materiales relativas a la sociedad, como la situación financiera y los resultados, siendo este un objetivo de las Normas Internacionales de Información financiera, las cuales buscan promover principios de transparencia que posteriormente se reflejan en los Estados Financieros y que sirven para la toma de decisiones de diversos usuarios.

Las continuas investigaciones en instituciones públicas asociadas al sector educativo, se ven reflejadas en el índice de transparencia anual de las entidades públicas, establecido por la Corporación Transparencia por Colombia que para el año 2013-2014, el cual arrojo que en promedio el índice de transparencia de las instituciones de
Educación Superior es del 56.4, tomando como referente una base de 0 a 100, mientras que la media en cuanto a divulgación de información asociada a gestión presupuestal y financiera es de 63, lo cual ubica a estas instituciones en un nivel de riesgo alto y en el mejor de los casos nivel de riesgo medio.

Dadas las anteriores condiciones sobre este grupo de instituciones se analizará el grado de cumplimiento frente a los criterios de presentación de la información contable para entidades del sector público, definidos por NICSP, considerando adicionalmente que la presentación de información financiera en estas entidades debe realizarse a partir del año 2018, siguiendo lo establecido en Resolución 533 de 2015, por medio de la cual estas entidades realizan convergencia hacia normas internacionales de contabilidad pública, pese a que ya desde el año 2007, con las resolución 354, 355 y 356, se realizó una adopción inicial de estándares internacionales de contabilidad para el sector público, situación que fue considera como un proceso de innovación para Colombia conforme a lo señalado por Gómez \& Montesinos (2012).

Considerando lo anterior se analizarán los

2 Organización para la cooperación y el desarrollo económico. 
Estados Financieros publicados en los portales web de las entidades educativas del sector público, identificando si cumplen con los criterios de presentación establecidos para los Estados Financieros, notas, y revelación de políticas contables, definidos para las entidades de gobierno en la Resolución 533 de 2015, conforme a lo establecido en las NICSP. Con el análisis realizado a los Estados Financieros publicados a 2016 por las instituciones objeto de estudio, será posible identificar cuáles son los principales factores que deben mitigar estas instituciones respecto a la información financiera a presentar en 2018, esto en razón a que la difusión de Estados Financieros y políticas Contables es un aspecto fundamental que debe optimizarse para mejorar los niveles de transparencia en la sociedad Colombiana tal y como lo ha establecido Baracaldo, (2013).

La población objeto de estudio está compuesta por 54 Instituciones de Educación Superior, entre las que se encuentran 47 universidades de orden departamental y municipal y 7 Instituciones técnicas y tecnológicas, para las cuales se realiza evaluación de los estados financieros publicados, con corte a diciembre de 2016, considerando que conforme a lo establecido por el comité interinstitucional de la comisión de estadísticas de finanzas públicas, dichas instituciones deben aplicar Normas Internacionales de Contabilidad para entidades del sector público, conforme a los criterios definidos en Resolución 533 de 2015 y sus anexos.

Con los datos obtenidos en el estudio, se identificarán los aspectos con menor grado de cumplimiento respecto a la presentación de Estados Financieros, políticas y notas de carácter específico, de las entidades de educación superior del sector público, para dichas entidades se esperan bajos niveles de cumplimiento en las revelaciones asociadas a las políticas contables, ya que siguiendo estudios previos realizados por Pérez y López (2003), los países Latinoamericanos tienen una tendencia menor a revelar las políticas contables de sus entidades, una situación que podría darse también para el caso Colombiano en las entidades objeto de análisis.

Por tal razón, en la investigación desarrollada se presentará primero la metodología empleada en el desarrollo del documento, posteriormente se presentarán los resultados obtenidos una vez aplicada la metodología seleccionada, para mostrar seguidamente las conclusiones del estudio. Finalmente, en el documento se presentaran los anexos con el análisis efectuado a cada uno de los factores de impacto determinados en la investigación.

\section{METODOLOGÍA, MATERIALES Y MÉTODO}

El índice de medición empleado, ha sido aplicado siguiendo en principio el modelo sugerido por Pérez \& López (2003), el cual ha sido actualizado en esta investigación considerando los cambios principales de las NICSP, en lo referente a los criterios de presentación de la información financiera y cuidando que ningún ítem fuese evaluado dos veces. En este sentido, se aplicó un primer índice de medición que evalúa, si las entidades objeto de análisis cumplen con la presentación de los estados financieros que requiere la NICSP sin importar si el contenido de los mismos está acorde a lo solicitado por la norma, para este primer índice se evaluaron seis aspectos que se detallan en la siguiente tabla 1.

Tabla 1. Índice de Cumplimiento $1\left(\mathrm{IC}_{1}\right)$.

\begin{tabular}{lc}
\hline \multicolumn{1}{c}{ Aspecto Evaluado (Estados Financieros, Políticas y Notas -EFPN-) } & Porcentaje \\
\hline Estado de situación Financiera o Balance General & $16,6 \%$ \\
Estado de Resultados & $16,6 \%$ \\
Cambios en los activos netos / Patrimonio Neto & $16,6 \%$ \\
Estado de Flujo de Efectivo & $16.6 \%$ \\
Políticas Contables & $16.6 \%$ \\
Notas Explicativas & $16.6 \%$ \\
\hline TOTAL & $100 \%$ \\
\hline
\end{tabular}

Fuente: Elaboración propia. 
En el segundo ítem de evaluación se consideraron 58 aspectos, en los cuales se analiza si la información presentada en cada uno de los Estados Financieros cumple con los criterios esta- blecidos en NICSP, estos elementos se presentan en forma detallada en los anexos presentados en esta investigación, los cuales se resumen a continuación:

Tabla 2. Índice de Cumplimiento $2\left(\mathrm{IC}_{2}\right)$.

\begin{tabular}{|c|c|c|}
\hline $\begin{array}{l}\text { Aspecto Evaluado (Componente individual presentados en } \\
\text { cada Estado Financiero) - CIPEF- }\end{array}$ & $\begin{array}{c}\text { Numero } \\
\text { de } \\
\text { Variables }\end{array}$ & Porcentaje \\
\hline Elementos del Estado de Situación Financiera & 17 & $29 \%$ \\
\hline Elementos del Estado de Resultados & 7 & $12 \%$ \\
\hline Elementos del Estado de Cambios en el Patrimonio & 6 & $10 \%$ \\
\hline Elementos del Estado de Flujo de Efectivo & 9 & $16 \%$ \\
\hline Elementos presentados en las Notas a los Estados Financieros & 6 & $10 \%$ \\
\hline $\begin{array}{l}\text { Elementos correspondientes a las políticas contables presenta- } \\
\text { das por la entidad }\end{array}$ & 13 & $23 \%$ \\
\hline TOTAL & 58 & $100 \%$ \\
\hline
\end{tabular}

Fuente: Elaboración propia.

El índice de cumplimiento se aplicó siguiendo el instrumento de medición sugerido por Pérez y López, (2003), en el cual se emplea una puntuación dicotómica, en donde si se cumple con el criterio evaluado se asigna una puntuación de uno, y cero si la entidad analizada no cumple con el aspecto evaluado. Por tanto, el índice de cumplimiento se medirá sumando el

$$
I C_{1}=\frac{\sum_{\text {Presentado }(\text { EFPN })}^{\text {Item efectivamente }}}{\text { Numero de Items a Evaluar }} \times 100
$$

En los aspectos evaluados para el segundo índice de cumplimiento es posible obtener medidas parciales, que permitan identificar el grado de cumplimiento en cada uno de los Estados Financieros, políticas contables y notas que deben presentar las entidades analizadas.

\section{RESULTADOS}

El análisis realizado a los estados financieros, presentados por las entidades de educación superior que deben aplicar Normas Internacionales de contabilidad para el sector público, reflejo número de aspectos que cumplen con la condición evaluada y dividiéndolo por el total de ítems evaluados, que para el primer índice de medición serian 6 aspectos evaluados, y para el segundo índice 58 aspectos evaluados.

Considerando lo anterior el índice de cumplimiento para cada grupo de factores evaluados estaría representado por:

$$
I C_{2}=\frac{\sum \begin{array}{c}
\text { Item efectivamente } \\
\text { Presentado }(\text { CIPEF })
\end{array}}{\text { Numero de Items a Evaluar }} \times 100
$$

que en el primer índice de cumplimiento que comprende la presentación de Estados financieros sin analizar su contenido, la media de cumplimiento es del $71 \%$, este porcentaje se debe en gran parte a que la mayoría de entidades no presentan el Estado de flujo de Efectivo, tan solo 6 de 48 Instituciones analizadas incluyeron dicho Estado Financiero, es decir un 13\% de las instituciones estudiadas, otro factor que afecta el índice de cumplimiento por la presentación de Estados Financieros, es el relacionado con las Políticas Contables, ya que solo un $65 \%$ de la población analizada revela información relacionada con sus políticas contables. 
Es importante precisar que al consultar la información financiera publicada en las páginas web de las entidades objeto de estudio, no fue posible obtener los Estados Financieros de 6 de las 54 entidades analizadas, pese que adicionalmente se solicitó la información de forma escrita, solo se obtuvo respuesta por parte una de las entidades analizadas, la cual informo que los Estados Financieros aun no podían ser publicados considerando que requerían aprobación del consejo superior de la universidad, en este aspecto es importante considerar que conforme lo establece la Resolución 354 de 2007, el plazo máximo de generación de información con corte a diciembre 31 de cada año, vence el 15 de febrero del periodo siguiente. Por lo cual a la fecha, más de 5 meses después del plazo máximo de reporte ya debería estar disponible la información financiera.

Para el caso de las seis entidades que no suministraron información respecto a sus estados financieros, las cuales se presentan en los anexos de esta investigación, se observó que las mismas se ubican en un nivel de riesgo alto o muy alto, conforme al índice de transparencia nacional divulgado por la organización transparencia por Colombia y aplicado a las entidades públicas de educación superior con corte al año 2014, el cual nos permite corroborar lo difícil que es obtener información de estas entidades, en donde pese a solicitud directa de la información la misma no fue entregada.

En cuanto al segundo índice, en donde se analizó si los Estados Financieros presentados cumplen con los criterios requeridos por NICSP el promedio de cumplimiento de las condiciones de presentación es del $46 \%$, para las 48 entidades analizadas, el mayor grado de cumplimiento alcanzado por una entidad es del $69 \%$ y el menor grado de cumplimiento es del $19 \%$, ya que en este segundo caso la entidad únicamente revelo información del Estado de Situación Financiera y del Estado de Resultados, la entidad no presento información relacionada con el Estado de Cambios en el patrimonio, Estado de Flujo de Efectivo, Notas a los Estados Financieros y políticas contables, una situación que se repite para algunas de las entidades analizadas.

Frente al Estado de situación Financiera, denominado por la resolución 354 de 2007, como Balance General, la media de cumplimiento en cuanto a los criterios de presentación establecidos por las Normas internacionales de Contabilidad aplicadas al sector público es del 80\%, para el caso en particular del análisis realizado al estado de situación financiera, tan solo una de las entidades estudiadas cumple con el 100\% de los aspectos establecidos en NICSP, y la entidad con menor grado de cumplimiento alcanza el 53\%, frente a los criterios evaluados.

En el caso del Estado de Resultados, una de las entidades analizadas apenas alcanza el $14 \%$ de cumplimiento frente a los criterios de presentación que deben considerarse para dicho estado financiero, conforme a lo establecido por NICSP, la media de cumplimiento en las entidades analizadas es del 69\%, y tan solo una de las entidades analizadas cumple con el $100 \%$ de los requisitos establecidos por las normas internacionales de contabilidad aplicadas al sector público.

Es importante precisar que la normatividad Colombiana denominaba al estado de resultados como Estado de Actividad financiera, económica, social y ambiental, este último diseñado bajo los paramentos contables públicos del año 2007, con los mismos fines previstos para el estado de resultados y con amplios grados de coincidencia frente a lo solicitado por NICSP.

Los resultados dados para el Estado de cambios en el patrimonio, nos permiten observar que la media en estas entidades en cuanto a los criterios de presentación que deben presentarse, alcanza apenas el $41 \%$, considerando que 14 de las 48 entidades analizadas no incluyen este estado financiero, entre el juego de Estados Financieros que son presentados con corte a 31 de diciembre de 2016, el porcentaje de cumplimiento más alto que alcanzan algunas de estas entidades es del $83 \%$, y en general la estructura de presentación del estado financiero no cumple con los requerimientos de NICSP.

En cuanto al Estado de Flujo de Efectivo, como mencionamos anteriormente tan solo 6 instituciones incluyen entre sus estados financieros el anteriormente mencionado, el porcentaje de cumplimiento más alto frente a los criterios presentados en este estado financiero, establecidos por Normas internacionales de contabilidad aplicadas al sector público es del 78\%, mientras que la institución con menor grado de cumplimiento refleja un porcentaje del $56 \%$.

Las políticas contables y las notas a los estados financieros, son uno de los factores con menor índice de cumplimiento para las entidades analizadas, algunas de las instituciones de educación superior estudiadas, cuentan con apenas un $5 \%$ de grado de cumplimiento frente a lo requerido por las NICSP, el porcentaje de cumplimiento más alto que logran algunas de las entidades analizadas es del $74 \%$, sin embargo tan solo seis de las entidades analizadas presentan índices que superen el 50\%, el resto de entidades 
presentan índices de cumplimiento entre el 5\% y el $47 \%$, un porcentaje considerablemente bajo.

\section{DISCUSIÓN, CONCLUSIONES}

Las entidades del sector público en Colombia están obligadas a revelar información de interés general para los diferentes usuarios de la misma, la Ley 1712 de marzo de 2014, establece la información mínima que debe revelarse en donde se incluye información asociada al presupuesto general y la ejecución presupuestal anual de estas entidades, sin embargo en la normatividad referida anteriormente, no se incluyó como información mínima a revelar la contenida en los estados financieros, un aspecto que debe considerarse por el gobierno nacional.

Las situaciones anteriormente previstas no implican que la información incluida en los estados financieros no se considere de carácter público, por lo cual debe estar disponible para los usuarios de la misma en los tiempos dispuestos por la contaduría General de la nación, entidad garante de la información contable publica en Colombia, por ello, resulta notable que para seis de las entidades analizadas no fuese posible obtener la información requerida, más aun si se considera el caso de la universidad del atlántico, en donde se informó que los estados financieros no habían sido publicados, dado que el consejo superior no los había aprobado, una situación que va en contravía de la normatividad colombiana puesto que el plazo máximo otorgado a estas entidades para presentar la información contenida en los estados financieros, venció en el mes de febrero de 2017.

La situación anterior, pone en evidencia el escaso control aplicado a estas entidades en cuanto a la revelación de información financiera, un aspecto que tiene un efecto directo en los índices de transparencia de las entidades analizadas, ya que el principio de transparencia converge como lo afirma Baracaldo, (2006) en la oportunidad de la información, la obtención de datos fidedignos y las revelaciones que deben generar confianza para los diversos grupos de interés de la información financiera. Una situación que no se cumple en los seis casos de las entidades mencionadas, ya que no existe oportunidad en la presentación de la información financiera por lo que se quebranta una de las características de la información financiera útil.

$\mathrm{Al}$ analizar la presentación de información financiera por parte de las entidades educativas del sector público que deben aplicar NICSP, se observa que existe un alto grado de incumplimiento frente a la presentación del Estado de Flujo de Efectivo, ya que la mayoría de entidades analizadas omiten su preparación, presentación y revelación. El estado de Flujo de efectivo conforme a lo establecido por Vargas, (2007), permite a los usuarios evaluar la capacidad de la entidad para generar efectivo e identificar las necesidades de liquidez, todo esto con el fin de tomar decisiones más optimas y razonables, ayudando a reducir la incertidumbre, mejorando la gestión empresarial, y permitiendo un control eficiente de los instrumentos financieros y las entradas y salidas de efectivo. De allí la importancia de que el estado financiero en mención sea incorporado, siendo este uno de los aspectos de mejora que deben observar este tipo de entidades al momento de aplicar estándares internacionales de contabilidad para el sector público.

De igual forma, pese a que la gran mayoría de entidades presentan el Estado de Cambios en el Patrimonio, el mismo no cumple con los criterios establecidos por las NICSP, por tanto la estructura de presentación deberá modificarse casi que por completo, para que allí se presenten los aspectos requeridos por los usuarios para la toma de decisiones financieras, como lo son los aumentos y disminuciones que afecten el capital y el efecto acumulado producido por los cambios en criterios contables y en la corrección de errores.

La revelación de políticas contables por parte de las entidades analizadas, conforman uno de los aspectos en los que deberán concentrarse los esfuerzos de estas entidades con el fin de aplicar NICSP, ya que como observamos el índice de cumplimiento es menor al 50\% para la mayoría de entidades analizadas, puesto que la revelación de políticas se centra en un análisis de cifras que no permiten identificar las características de reconocimiento y los modelos de medición que aplica la entidad, siendo semejante la información presentada en las notas a los estados financieros y en las políticas contables, por lo que dichas políticas no cumplen con los requerimientos establecidos en NICSP, o son inexistentes para algunas de las entidades analizadas, razón por la cual es importante considerar que las organizaciones deben llevar contabilidad no para cumplir con un requisito legal, si no para presentar información confiable y fidedigna que sirva para la toma de decisiones por parte de los usuarios (Chacón, 2006), una tarea en la que resulta fundamental la correcta elaboración de no- 
tas a los estados financieros y políticas contables.

Considerando lo anterior se evidencia que las similitudes más grandes frente a los criterios de presentación requeridos por NICSP y la normatividad contable colombiana aplicada a las entidades del Sector Publico, se dan en aspectos relacionados con el Estado de Situación financiera y el Estado de resultados, aunque ambos bajo normatividad local estén denominados en forma diferente. En cuanto a los aspectos a modificar, se deben priorizar los ajustes al Estado de Cambios en el Patrimonio ya que el mismo no refleja la información requerida por NICSP. Frente al Estado de Flujo de efectivo, la mayoría de entidades deben iniciar su preparación, considerando que muy pocas instituciones presentan dicho estado financiero. De igual forma, las entidades objeto de análisis deben trabajar en la elaboración de políticas contables adecuadas y en la correcta presentación de las notas a los estados financieros, que en algunos casos son omitidas cuando se presentan los estados financieros.

Finalmente, producto del estudio realizado se observa que una de las principales falencias de estas entidades se da en la elaboración de políticas contables acorde a lo requerido por NICSP, generándose en este campo una nueva línea de estudio que permita identificar de manera detallada la importación de las políticas contables, los efectos de las políticas contables en los estados financieros y los aspectos claves que deben considerarse en la estructuración de las mismas.

\section{REFERENCIAS BIBLIOGRÁFICAS}

Baracaldo, N. (2013). ¿Es garante del principio de transparencia la implementación de Normas Internacionales en Colombia? Revista Cuadernos de Contabilidad, 14(36), pp. 1097-1120.

Chacón, G. (2006). Relevancia de la información contable. Revista actualidad contable Faces, volumen 9, número 13, Julio - Diciembre de 2016, pp. 3-4.

Contaduría General de la Nación, (2016). Entidades sujetas al ámbito de aplicación de la Resolución 533 de 2015. Régimen de contabilidad pública, marco normativo para entidades de gobierno, comité interinstitucional de la comisión de estadísticas de finanzas públicas pp. 1-46.
Contaduría General de la Nación, (2007). Resolución 354 "Por el cual se adopta el Régimen de Contabilidad Pública, se establece su conformación y se define el ámbito de aplicación".

Contaduría General de la Nación, (2013) Resolución 743. "Por la cual se incorpora el en el Régimen de Contabilidad Pública, el marco normativo aplicable para algunas empresas sujetas a su ámbito y se dictan otras disposiciones".

Contaduría General de la Nación, (2015) Resolución 533. "Por la cual se incorpora, en el Régimen de contabilidad pública, el marco normativo aplicable a entidades de gobierno y se dictan otras disposiciones".

Congreso de la Republica de Colombia (2014). Por medio de la cual se crea la Ley de Transparencia y del Derecho de Acceso a la Información Pública Nacional y se dictan otras disposiciones. Ley 1712 de 2014.

Corporación transparencia por Colombia (2015). Colección de documentos observatorio de integridad No. 16, índice de transparencia nacional 2013-2014.

Gómez, M. \& Montesinos, V., (2012). Las innovaciones en contabilidad gubernamental en Latinoamérica: el caso de Colombia. Innovar, 22(45), 17-35.

Johnston, D., (2004). Principios de gobierno corporativo, Organización para la cooperación y el desarrollo Económico.

Pérez, C. \& López, A., (2003). La difusión de información financiera gubernamental en los países del Mercosur: Su amortización a través de la aplicación de IPSAS de la IFAC. Revista contabilidad y finanzas - USP, Vol. 14, núm. 33, pp. 90-100.

Vargas, R. (2007). Estado de Flujo de Efectivo. Revista Intersedes. Costa Rica, Volumen VIII, número 14 de 2007, pp. 111-136. 
ANEXO 1.

Total de puntuación obtenida por cada uno de los conceptos analizados para el índice de cumplimiento 1 y 2.

\begin{tabular}{|c|c|c|c|}
\hline $\begin{array}{l}\text { Número } \\
\text { asignado }\end{array}$ & Institución de educación superior que deben aplicar NICSP & $\begin{array}{c}\text { Índice de } \\
\text { Cumplimiento } \\
1\end{array}$ & $\begin{array}{c}\text { Índice de } \\
\text { Cumplimiento } \\
2\end{array}$ \\
\hline 1 & Colegio Mayor de Antioquia & $83 \%$ & $53 \%$ \\
\hline 2 & Colegio Mayor de Bolívar & $67 \%$ & $43 \%$ \\
\hline 3 & Colegio Mayor del Cauca & $83 \%$ & $43 \%$ \\
\hline 4 & Escuela Superior de Administración Pública & $67 \%$ & $41 \%$ \\
\hline 5 & Escuela Tecnológica Instituto Técnico Central & $83 \%$ & $36 \%$ \\
\hline 6 & Institución Universitaria Antonio José Camacho & $100 \%$ & $47 \%$ \\
\hline 7 & Institución universitaria de Envigado & $100 \%$ & $59 \%$ \\
\hline 8 & Instituto de Educación Técnica Profesional de Roldanillo & $50 \%$ & $40 \%$ \\
\hline 9 & Instituto Superior de Educación Rural de Pamplona & $33 \%$ & $19 \%$ \\
\hline 10 & Instituto Tecnológico de Soledad Atlántico & $83 \%$ & $41 \%$ \\
\hline 11 & Instituto Tecnológico del Putumayo & $67 \%$ & $33 \%$ \\
\hline 12 & Instituto Tecnológico Metropolitano & $67 \%$ & $45 \%$ \\
\hline 13 & Instituto Tolimense de Formación Técnica Profesional & $83 \%$ & $50 \%$ \\
\hline 14 & Instituto Universitario de la Paz & $83 \%$ & $45 \%$ \\
\hline 15 & Politécnico Colombiano Jaime Isaza Cadavid & $67 \%$ & $40 \%$ \\
\hline 16 & Universidad Central del Valle del Cauca & $83 \%$ & $43 \%$ \\
\hline 17 & Universidad Colegio Mayor de Cundinamarca & $50 \%$ & $33 \%$ \\
\hline 18 & Universidad de Antioquia & $83 \%$ & $53 \%$ \\
\hline 19 & Universidad de Caldas & $67 \%$ & $48 \%$ \\
\hline 20 & Universidad de Cartagena & $67 \%$ & $41 \%$ \\
\hline 21 & Universidad de Cundinamarca & $33 \%$ & $36 \%$ \\
\hline 22 & Universidad de la Amazonía & $83 \%$ & $52 \%$ \\
\hline 23 & Universidad de la Guajira & $50 \%$ & $40 \%$ \\
\hline 24 & Universidad de los Llanos & $83 \%$ & $41 \%$ \\
\hline 25 & Universidad de Nariño & $67 \%$ & $47 \%$ \\
\hline 26 & Universidad de Pamplona & $67 \%$ & $57 \%$ \\
\hline 27 & Universidad de Sucre & $50 \%$ & $36 \%$ \\
\hline 28 & Universidad del Cauca & $50 \%$ & $38 \%$ \\
\hline 29 & Universidad del Magdalena & $33 \%$ & $36 \%$ \\
\hline 30 & Universidad del Pacífico & $100 \%$ & $57 \%$ \\
\hline 31 & Universidad del Quindío & $33 \%$ & $36 \%$ \\
\hline 32 & Universidad del Tolima & $83 \%$ & $50 \%$ \\
\hline 33 & Universidad del Valle & $100 \%$ & $69 \%$ \\
\hline 34 & Universidad Distrital Francisco José de Caldas & $83 \%$ & $57 \%$ \\
\hline
\end{tabular}


35 Universidad Francisco de Paula Santander - seccional Ocaña

$\begin{array}{ll}33 \% & 31 \% \\ 83 \% & 59 \% \\ 83 \% & 52 \% \\ 83 \% & 62 \% \\ 100 \% & 60 \% \\ 50 \% & 36 \% \\ 83 \% & 50 \% \\ 50 \% & 45 \% \\ 67 \% & 45 \% \\ 83 \% & 52 \% \\ 50 \% & 40 \% \\ 83 \% & 45 \% \\ 100 \% & 53 \% \\ 83 \% & 62 \%\end{array}$

36 Universidad Militar Nueva Granada

$\%$

37 Universidad Nacional Abierta y a Distancia (6)

38 Universidad Nacional de Colombia

39 Universidad Pedagógica Nacional

40 Universidad Pedagógica y Tecnológica de Colombia

41 Universidad Popular del Cesar

42 Universidad Surcolombiana

43 Universidad Tecnológica de Pereira

44 Universidad Tecnológica del Chocó Diego Luis Córdoba

45 Colegio Integrado Nacional Oriente de Caldas

46 Instituto Nacional de Formación Técnica Profesional de San Juan del Cesar

47 Instituto Técnico Nacional de Comercio Simón Rodriguez

48 Servicio Nacional de Aprendizaje

$62 \%$

ANEXO 2.

Instituciones de Educación Superior que no aportaron información respecto a sus Estados Financieros.

\begin{tabular}{l}
\hline \multicolumn{1}{c}{ Institución de educación superior que debe aplicar NICSP (Sin Información) } \\
\hline Universidad de Atlántico \\
Universidad Francisco de Paula Santander - seccional Cúcuta \\
Universidad Industrial de Santander \\
Centro Tecnológico de Cúcuta \\
Instituto Nacional de Formación Técnica Profesional de San Andrés y Providencia \\
Instituto Nacional de Formación Técnica Profesional Humberto Velásquez GARCIA \\
\hline
\end{tabular}

ANEXO 3.

Conceptos Evaluados para el índice de cumplimiento No. 2.

\section{ELEMENTOS PRESENTADOS EN EL CUERPO DEL BALANCE GENERAL}

1 Activos no financieros (Inmovilizado no Financiero)

2 Activos Tangibles bienes muebles e inmuebles (Inmovilizado Material).

3 Activos Intangibles (Inmovilizado inmaterial)

4 Inversiones Financieras

5 Presentar en forma separada inversiones a largo plazo de las de corto plazo

6 Deudores

7 Mostrar separadamente los deudores de Largo plazo y los de corto Plazo

8 Efectivo y otros medios líquidos equivalentes

9 Anticipos 
10 Acreedores

11 Mostrar separadamente los acreedores de Largo plazo y los de corto Plazo

12 Prestamos

13 Mostrar separadamente los préstamos de largo plazo de los de corto plazo

14 Mostrar separadamente la porción de préstamos a largo plazo con vencimiento a corto plazo

15 Provisiones

16 Patrimonio

17 Distinción de los activos y pasivos conforme a su función de liquidez (Circulantes y no circulantes)

\section{ELEMENTOS PRESENTADOS EN EL CUERPO DEL ESTADO DE RESULTADOS}

18 Discrimina separadamente Ingresos con contraprestación

19 Discrimina separadamente Ingresos sin contraprestación

20 Los gastos son agregados de acuerdo con su naturaleza o su función

21 Se discriminan los gastos Financieros

22 Déficit o Superávit de actividades ordinarias

23 Déficit o Superávit de actividades Extraordinarias

24 Déficit o Superávit Neto de Periodo

\section{ELEMENTOS PRESENTADOS EN EL ESTADO DE CAMBIOS EN EL PATRIMONIO}

25 Conciliación entre el valor inicial y final en cada periodo de cada uno de los componentes del patrimonio

26 Déficit o superávit neto del periodo

27 Cada una de las partidas que se abonen o carguen directamente al patrimonio y el total de estos elementos

28 El efecto acumulado de los cambios en los criterios contables y en la corrección de errores

29 El saldo de los déficits o superávit acumulados al principio del periodo y en la fecha de cierre del balance y los movimientos del periodo

30 El resultado del periodo mostrando en forma separada, los importes totales atribuibles a las participaciones no controladas y a la entidad controladora

ELEMENTOS PRESENTADOS EN EL CUERPO DEL ESTADO DE FLUJO DE EFECTIVO

31 Mostrar separadamente el uso neto de caja proveniente de actividades de operación.

32 Mostrar separadamente las principales partidas de caja recibida y entregada por actividades de operación

33 Mostrar separadamente el uso neto de caja proveniente de actividades de inversión

34 Mostrar separadamente las principales partidas de caja recibida y entregada por actividades de inversión

35 Mostrar separadamente el uso neto de caja proveniente de actividades de financiación

36 Mostrar separadamente las principales partidas de caja recibida y entregada por actividades de financiación

37 Mostrar separadamente el flujo de caja asociado a actividades extraordinarias

38 Mostrar separadamente el flujo de caja asociado a intereses recibidos y pagados tanto por actividades de operación como de inversión y financiación. 
39 Mostrar una reconciliación entre las cantidades que se muestran en el flujo de efectivo y las que se muestran en el balance de situación

INFORMACIÓN DE APOYO PRESENTADA EN NOTAS A LOS ESTADOS FINANCIEROS

40 Efectivo no disponible para ser utilizado por la entidad

41 Contingencias no incluidas en el Balance

42 Compromisos no incluidos en el Balance

43 Información no financiera no incluida en el Balance

44 Referencia cruzada que permita la identificación de cada nota

45 Base de medida utilizada

\section{PRESENTACIÓN DE POLÍTICAS CONTABLES ESPECIFICAS PARA:}

46 Reconocimiento de los ingresos

47 Principios de Consolidación

48 Inversiones Financieras

49 Reconocimiento y depreciación de los activos tangibles e intangibles

50 Capitalización de los gastos financieros y otros gastos

51 Contratos de Construcción

52 Inversiones Inmobiliarias

53 Inversiones e instrumentos financieros

54 Provisiones para pensiones

55 Gastos de Investigación y desarrollo

56 Costes de pensiones por jubilaciones

57 Moneda Extranjera

58 Contabilidad en épocas de inflación

\section{ANEXO 4.}

Listado de instituciones evaluadas.

1. Colegio Mayo de Antioquia, (2017). Estados Financieros a Diciembre de 2016. Recuperado de http:/ / www.colmayor.edu.co/

2. Colegio Mayor de Bolívar, (2017). Estados Financieros a Diciembre de 2016. Recuperado de http:/ / www.colmayorbolivar.edu.co/

3. Colegio Mayor de Cauca, (2017). Estados Financieros a Diciembre de 2016. Recuperado de http: / / www.unimayor.edu.co/

4. Colegio Integrado Nacional Oriente de Caldas, (2017). Estados Financieros a Diciembre de 2016. Recuperado de https: / / wp.iescinoc.edu.co/

5. Escuela Superior de administración pública, (2017). Estados Financieros a diciembre de 2016. Recuperado de http: / / www.esap.edu.co/

6. Escuela Tecnológico Instituto Técnico Central, (2017). Estados Financieros a diciembre de 2016. Recuperado de http: / / www.itc.edu.co/

7. Institución Universitaria Antonio José Camacho, (2017). Estados Financieros a diciembre de 2016. Recuperado de http: / / www.uniajc.edu.co/

8. Institución universitaria de Envigado, (2017). Estados Financieros a diciembre de 2016. Recuperado de http:/ / www.iue.edu.co/

9. Instituto de Educación Técnica Profesional de Roldanillo, (2017). Estados Financieros a diciembre de 2016. Recuperado de http: / / www.intep.edu.co/

10. Instituto Nacional de Formación Técnica Profesional de San Juan del Cesar, (2017). Estados Fi- 
nancieros a diciembre de 2016. Recuperado de http: / / www.infotep.edu.co/

11. Instituto Superior de Educación Rural de Pamplona, (2017). Estados Financieros a diciembre de 2016. Recuperado de http: / / www.iser.edu.co/

12. Instituto Técnico Nacional de Comercio Simón Rodriguez, (2017). Estados Financieros a diciembre de 2016. Recuperado de http: / / www.intenalco.edu.co/

13. Instituto Tecnológico de Soledad Atlántico, (2017). Estados Financieros a diciembre de 2016. Recuperado de http: / / www.itsa.edu.co/

14. Instituto Tecnológico del Putumayo, (2017). Estados Financieros a diciembre de 2016. Recuperado de http:/ / www.itp.edu.co/

15. Instituto Tecnológico Metropolitano, (2017). Estados Financieros a diciembre de 2016. Recuperado de http:/ / www.itm.edu.co/

16. Instituto Tolimense de Formación Técnica Profesional, (2017). Estados Financieros a diciembre de 2016. Recuperado de http: / / itfip.edu.co/

17. Instituto Universitario de la Paz, (2017). Estados Financieros a diciembre de 2016. Recuperado de http://www.unipaz.edu.co/

18. Politécnico Colombiano Jaime Isaza Cadavid, (2017). Estados Financieros a diciembre de 2016. Recuperado de http: / / www.politecnicojic.edu.co/

19. Servicio Nacional de Aprendizaje, (2017). Estados Financieros a diciembre de 2016. Recuperado de http:/ / www.sena.edu.co/

20. Universidad Central del Valle del Cauca, (2017). Estados Financieros a diciembre de 2016. Recuperado de http: / / www.uceva.edu.co/

21. Universidad Colegio Mayor de Cundinamarca, (2017). Estados Financieros a diciembre de 2016. Recuperado de http: / / www.unicolmayor.edu.co/

22. Universidad de Antioquia, (2017). Estados Financieros a diciembre de 2016. Recuperado de http: / / www.udea.edu.co/

23. Universidad de Caldas, (2017). Estados Financieros a diciembre de 2016. Recuperado de http:/ / sig.ucaldas.edu.co/

24. Universidad de Cartagena, (2017). Estados Financieros a diciembre de 2016. Recuperado de http: / / www.unicartagena.edu.co/

25. Universidad de Cundinamarca, (2017). Estados Financieros a diciembre de 2016. Recuperado de https: / / www.ucundinamarca.edu.co/

26. Universidad de la Amazonía, (2017). Estados Financieros a diciembre de 2016. Recuperado de http: / / www.uniamazonia.edu.co/

27. Universidad de la Guajira, (2017). Estados Financieros a diciembre de 2016. Recuperado de http:/ / www.uniguajira.edu.co/

28. Universidad de los Llanos, (2017). Estados Financieros a diciembre de 2016. Recuperado de http: / / www.unillanos.edu.co/

29. Universidad de Nariño, (2017). Estados Financieros a diciembre de 2016. Recuperado de http:/ / www2.udenar.edu.co/

30. Universidad de Pamplona, (2017). Estados Financieros a diciembre de 2016. Recuperado de http: / / www.unipamplona.edu.co/

31. Universidad de Sucre, (2017). Estados Financieros a diciembre de 2016. Recuperado de http:/ / www.unisucre.edu.co/

32. Universidad del Cauca, (2017). Estados Financieros a diciembre de 2016. Recuperado de http:/ / www.unicauca.edu.co/

33. Universidad del Magdalena, (2017). Estados Financieros a diciembre de 2016. Recuperado de http: / / ciudadano.unimagdalena.edu.co /

34. Universidad del Pacífico, (2017). Estados Financieros a diciembre de 2016. Recuperado de http:/ / www.unipacifico.edu.co

35. Universidad del Quindío, (2017). Estados Financieros a diciembre de 2016. Recuperado de https: / / www.uniquindio.edu.co/

36. Universidad del Tolima, (2017). Estados Financieros a diciembre de 2016. Recuperado de http:/ / administrati.ut.edu.co/

37. Universidad del Valle, (2017). Estados Financieros a diciembre de 2016. Recuperado de http:/ / www.univalle.edu.co/ 
38. Universidad Distrital Francisco José de Caldas, (2017). Estados Financieros a diciembre de 2016. Recuperado de http: / / www.udistrital.edu.co

39. Universidad Francisco de Paula Santander - seccional Ocaña, (2017). Estados Financieros a diciembre de 2016. Recuperado de https:/ / ufpso.edu.co/

40. Universidad Militar Nueva Granada, (2017). Estados Financieros a diciembre de 2016. Recuperado de http:/ / www.umng.edu.co/

41. Universidad Nacional Abierta y a Distancia, (2017). Estados Financieros a diciembre de 2016. Recuperado de https: / / gaf.unad.edu.co/

42. Universidad Nacional de Colombia, (2017). Estados Financieros a diciembre de 2016. Recuperado de http:/ / gerencia.unal.edu.co/

43. Universidad Pedagógica Nacional, (2017). Estados Financieros a diciembre de 2016. Recuperado de http:/ / www.pedagogica.edu.co/

44. Universidad Pedagógica y Tecnológica de Colombia, (2017). Estados Financieros a diciembre de 2016. Recuperado de http: / / www.uptc.edu.co/

45. Universidad Popular del Cesar, (2017). Estados Financieros a diciembre de 2016. Recuperado de http:/ / www.unicesar.edu.co/

46. Universidad Surcolombiana, (2017). Estados Financieros a diciembre de 2016. Recuperado de https: / / www.usco.edu.co/

47. Universidad Tecnológica de Pereira, (2017). Estados Financieros a diciembre de 2016. Recuperado de http: / / www.utp.edu.co

48. Universidad Tecnológica del Chocó Diego Luis Córdoba, (2017). Estados Financieros a diciembre de 2016. Recuperado de https: / / www.utch.edu.co / 